Supporting Information

\title{
Constructing of Efficient Single Rh Sites on Activated Carbon via Surface Carbonyl Group for Methanol Carbonylation
}

Siquan Feng ${ }^{1}$, Xiangsong Lin $^{2 *}$, Xiangen Song ${ }^{*}$, Bingbao, $\mathrm{Mei}^{3}$, Jiali $\mathrm{Mu}^{1}$, Jingwei $\mathrm{Li}^{1}$, Yang Liu ${ }^{3,4}$, Zheng Jiang ${ }^{3}$, Yunjie Ding ${ }^{1,5^{*}}$

${ }^{1}$ Dalian National Laboratory for Clean Energy, Dalian Institute of Chemical Physics, Chinese Academy of Sciences, Dalian, China

${ }^{2}$ School of materials and textile Engineering, Jiaxing University, Jiaxing, Zhejiang, China

${ }^{3}$ Shanghai Synchrotron Radiation Facility, Shanghai Institute of Applied Physics; shanghai Advanced Research Institute, Chinese Academy of Sciences, Shanghai, China

${ }^{4}$ University of Chinese Academy of Sciences, Beijing, China

${ }^{5}$ State Key Laboratory of catalysis, Dalian Institute of Chemical Physics, Chinese Academy of Sciences, Dalian, China

Corresponding Authors:

Yunjie Ding (dyj@dicp.ac.cn); Xiangen Song (xiangensong@dicp.ac.cn);

Xiangsong Lin (xslin@dicp.ac.cn). 


\section{Table of Content}

\section{Supplementary Figures}

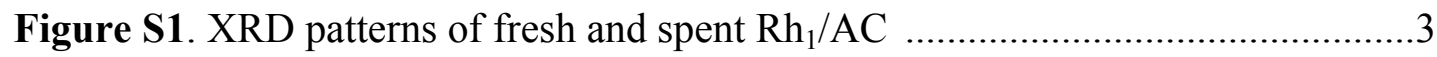

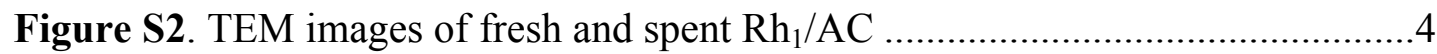

Figure S3. SEM of $\mathrm{Rh}_{1} / \mathrm{AC}$ and corresponding EDS mapping ................................5

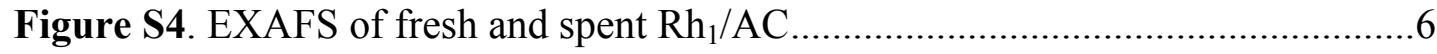

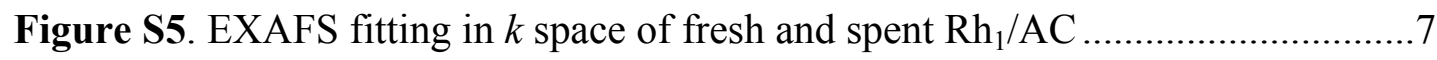

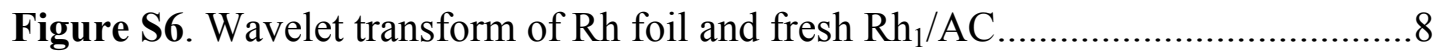

Figure S7. ATR-FTIR of fresh and spent $\mathrm{Rh}_{1} / \mathrm{AC}$ catalyst ....................................

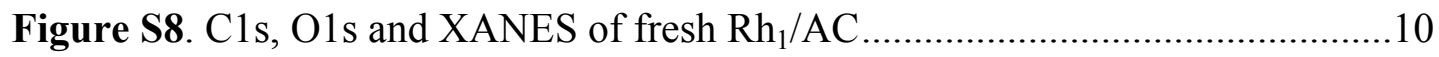

Figure S9. Thermal stability of oxygen-containing groups on AC support ..............11

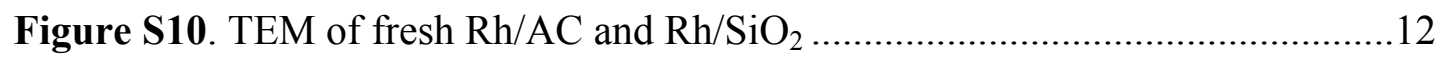

Figure S11. DFT calculation of homogeneous methanol carbonylation process ......13

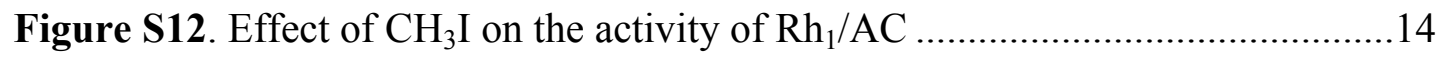

\section{Supplementary Tables}

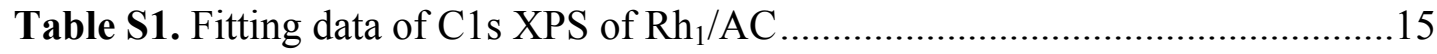

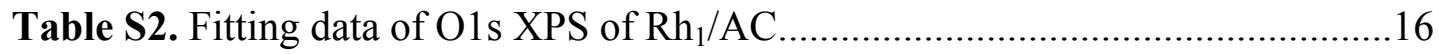

Table S3. Activity comparison between $\mathrm{Rh}_{1} / \mathrm{AC}$ and other catalysts .....................17

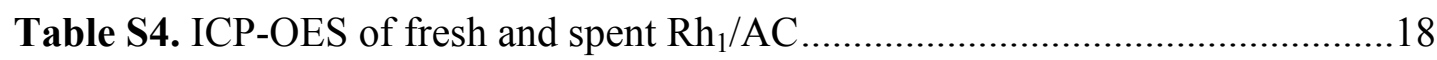

Table S5. Activity comparison of $\mathrm{Rh}_{1} / \mathrm{AC}, \mathrm{Rh} / \mathrm{SiO}_{2}$, and $\left[\mathrm{Rh}(\mathrm{CO})_{2} \mathrm{I}_{2}\right]^{-} \ldots \ldots \ldots \ldots \ldots \ldots . . . .19$

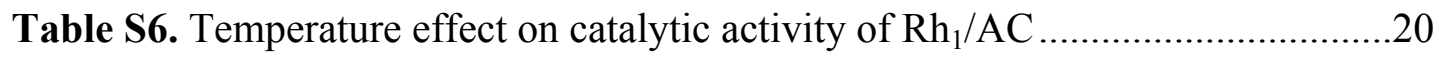

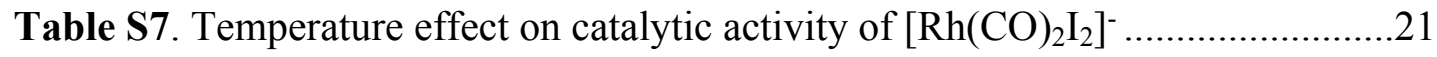

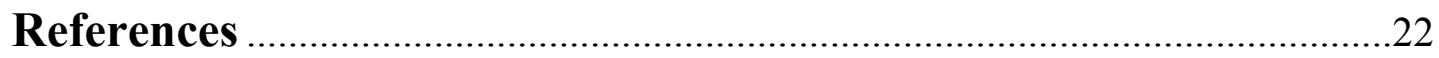




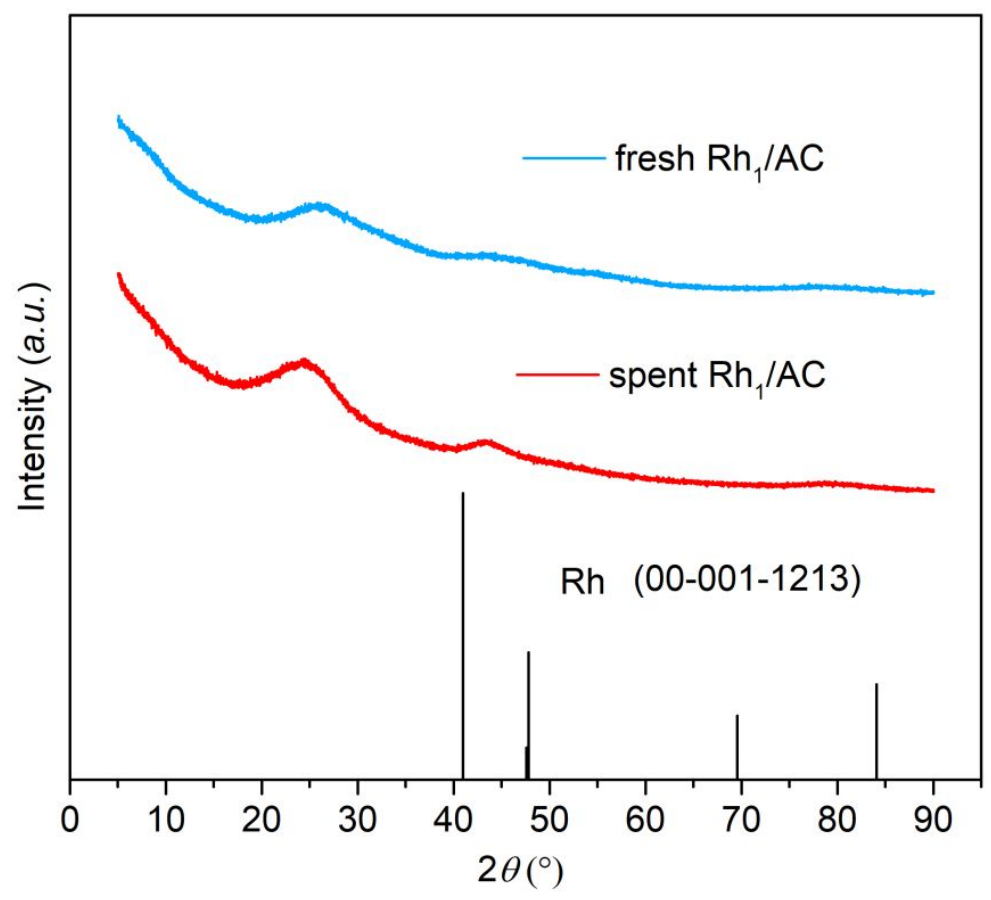

Figure S1. XRD patterns of fresh and spent $\mathrm{Rh}_{1} / \mathrm{AC}$ catalysts. The characteristic peak around $26^{\circ}$ in $\mathrm{XRD}$ patterns should be attributed to the graphitization carbon peak

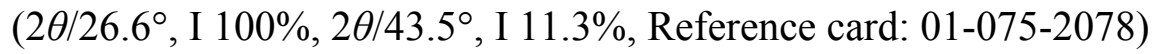


a

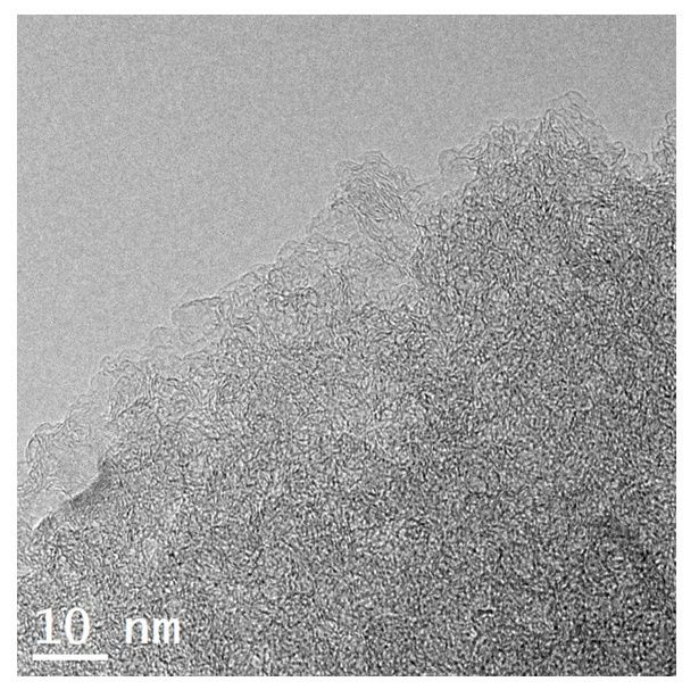

b

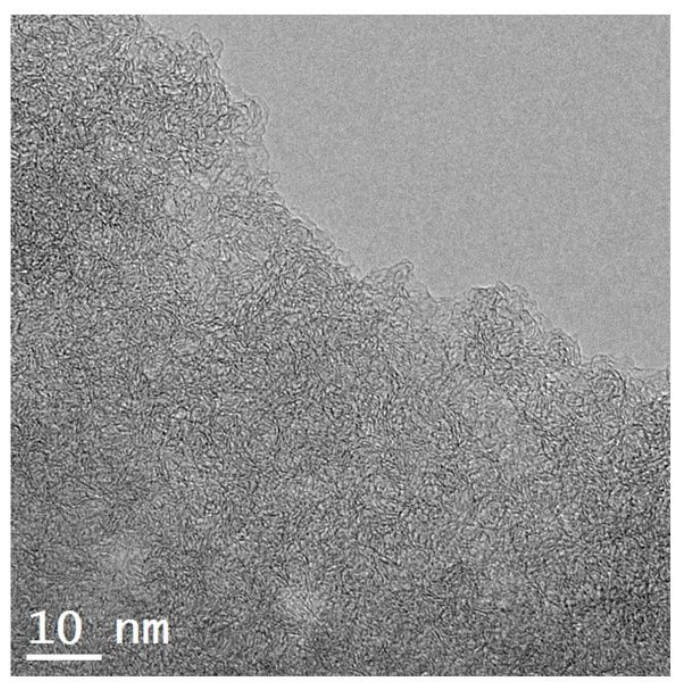

Figure S2. TEM images of (a) fresh and (b) spent $\mathrm{Rh}_{1} / \mathrm{AC}$ catalysts. 

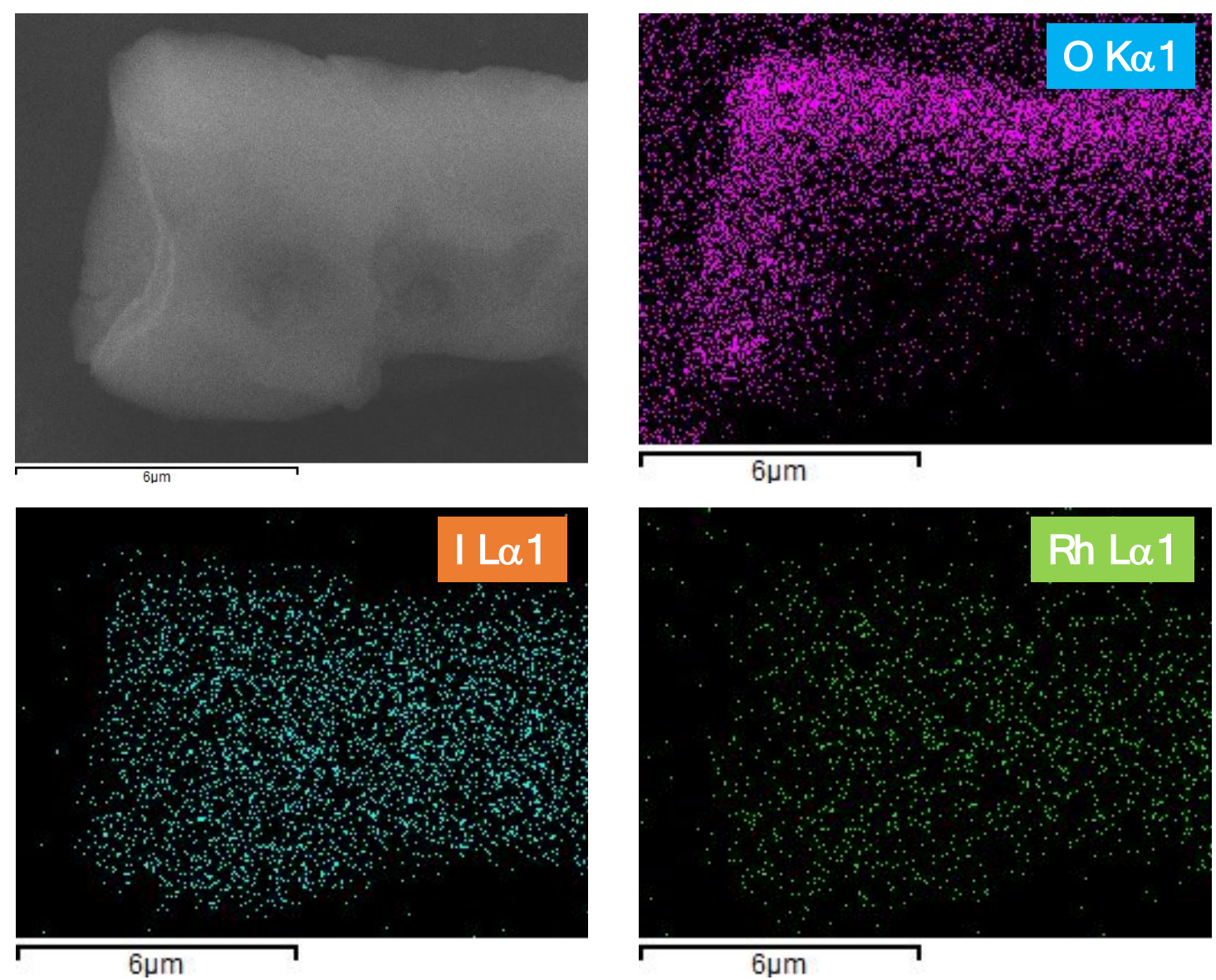

$6 \mu \mathrm{m}$

$6 \mu \mathrm{m}$

Figure S3. The pictures of $\mathrm{SEM}$ for $\mathrm{Rh}_{1} / \mathrm{AC}$ catalyst and corresponding EDS mapping of $\mathrm{O}$, I, and Rh elements. 
a

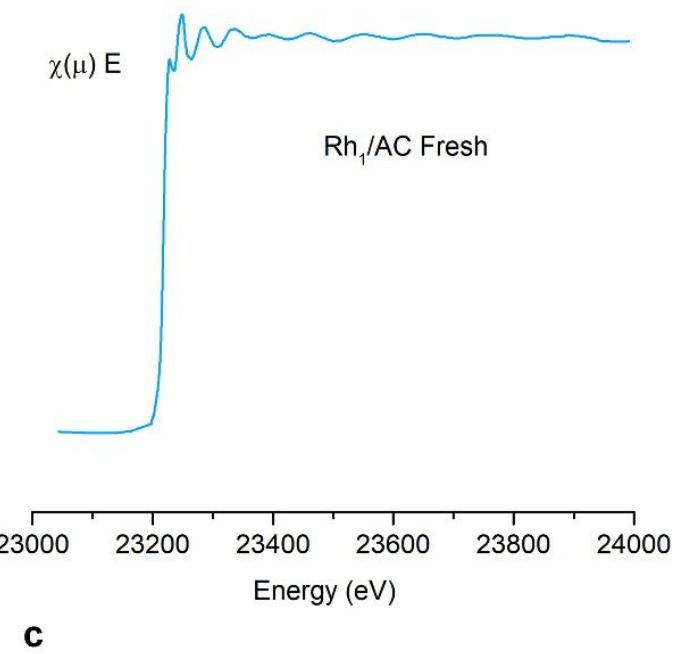

C

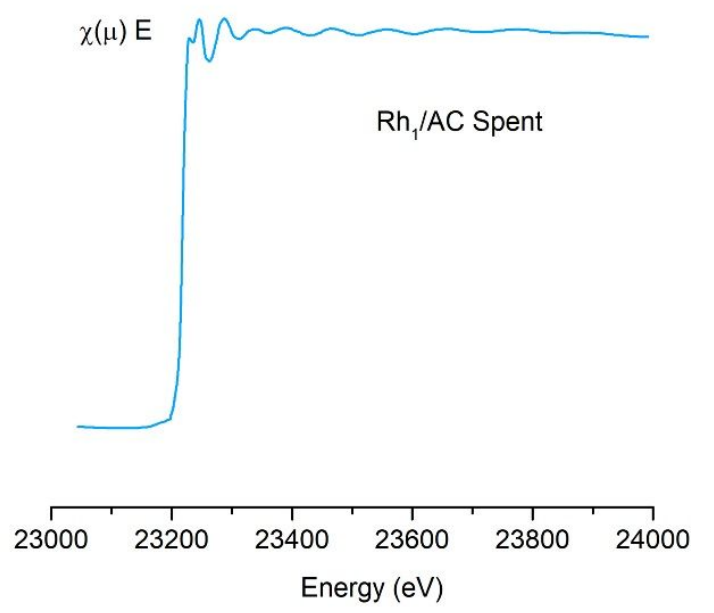

b
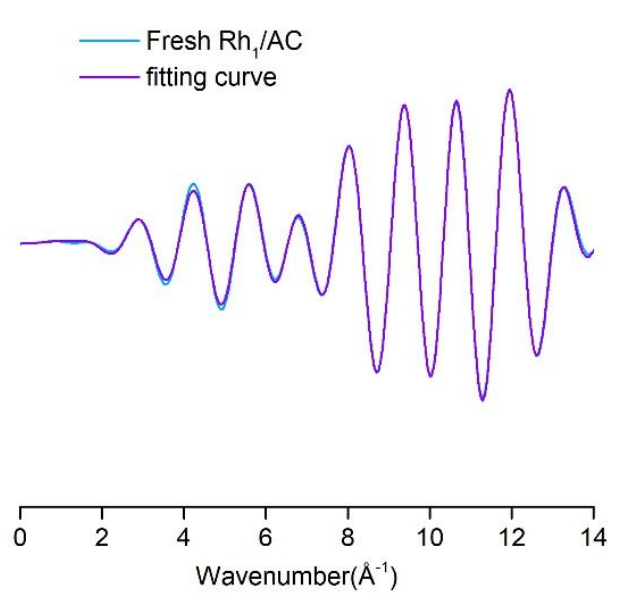

d

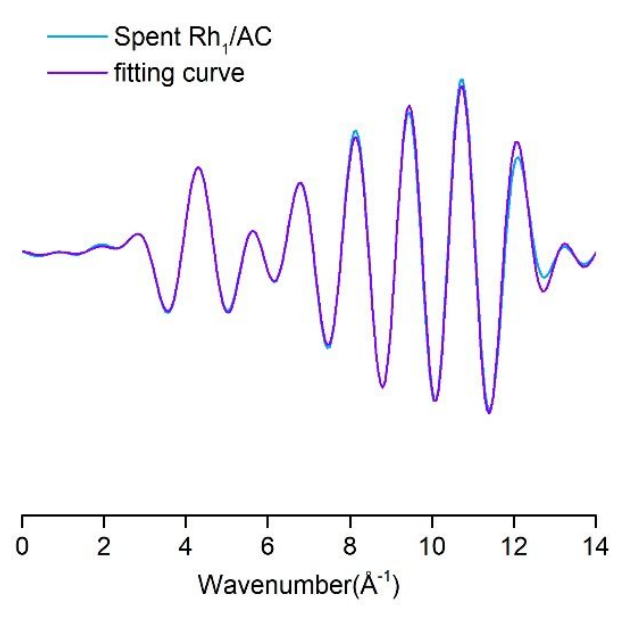

Figure S4. EXAFS data and the corresponding fitting in q space of (a \& b) fresh $\mathrm{Rh}_{1} / \mathrm{AC}$ and $(\mathbf{c} \& \mathbf{d})$ spent $\mathrm{Rh}_{1} / \mathrm{AC}$ catalysts for methanol carbonylation. 
a

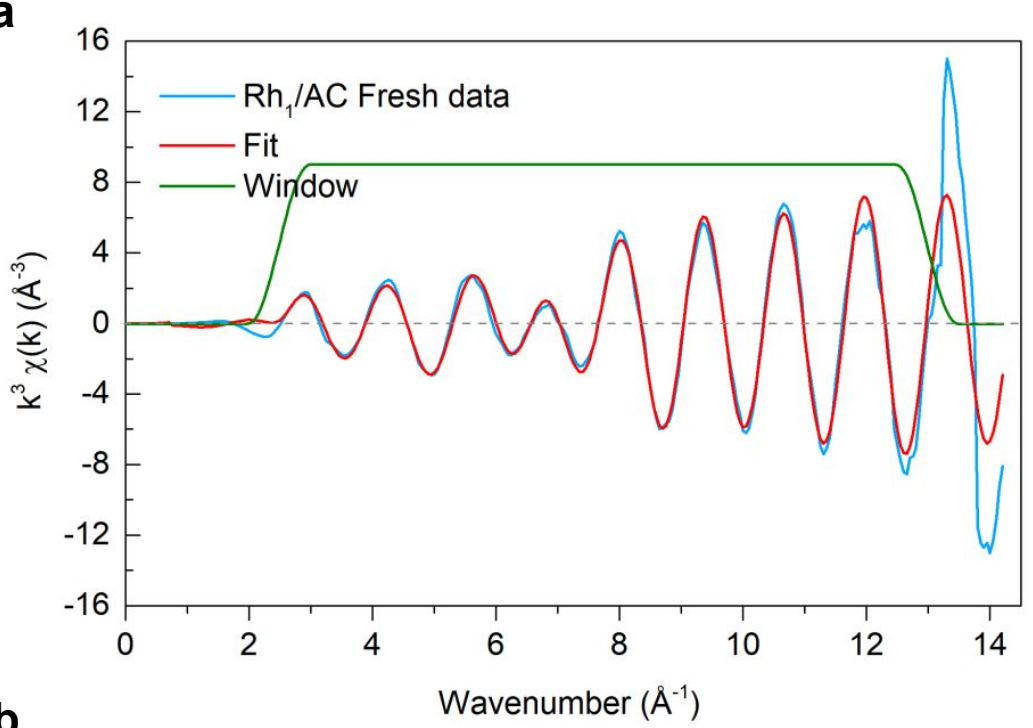

b

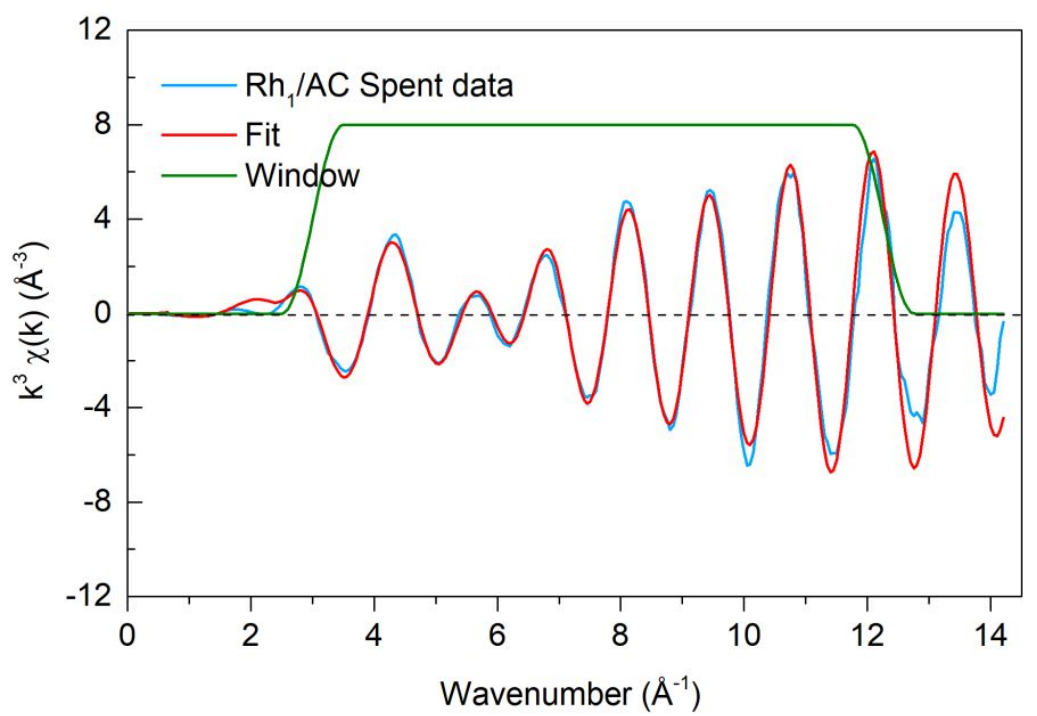

Figure S5. The EXAFS fitting plots and spectra in $k$ space of $\mathrm{Rh}_{1} / \mathrm{AC}$ fresh and spent catalysts. 

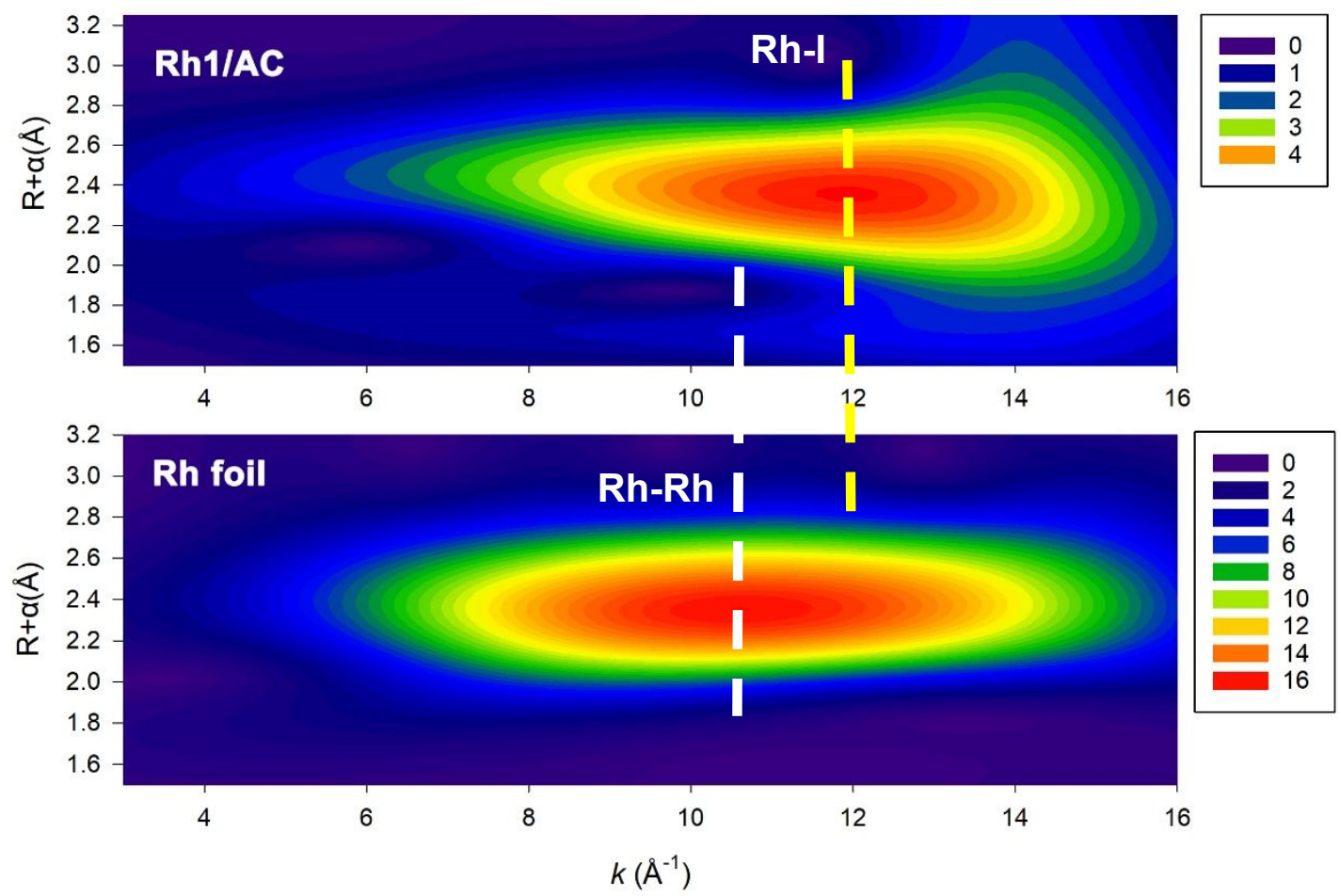

Figure S6. The wavelet transform contour plots of $k^{3}$-weighted $\chi(\mathrm{k})$ EXAFS signals of $\mathrm{Rh}$ foil and fresh $\mathrm{Rh}_{1} / \mathrm{AC}$ catalyst. 
$\mathrm{Rh}_{1} / \mathrm{AC}$ fresh

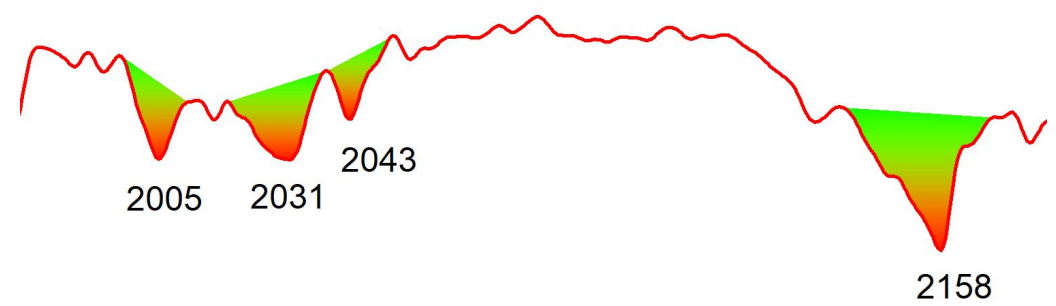

$\mathrm{Rh}_{1} / \mathrm{AC}$ spent

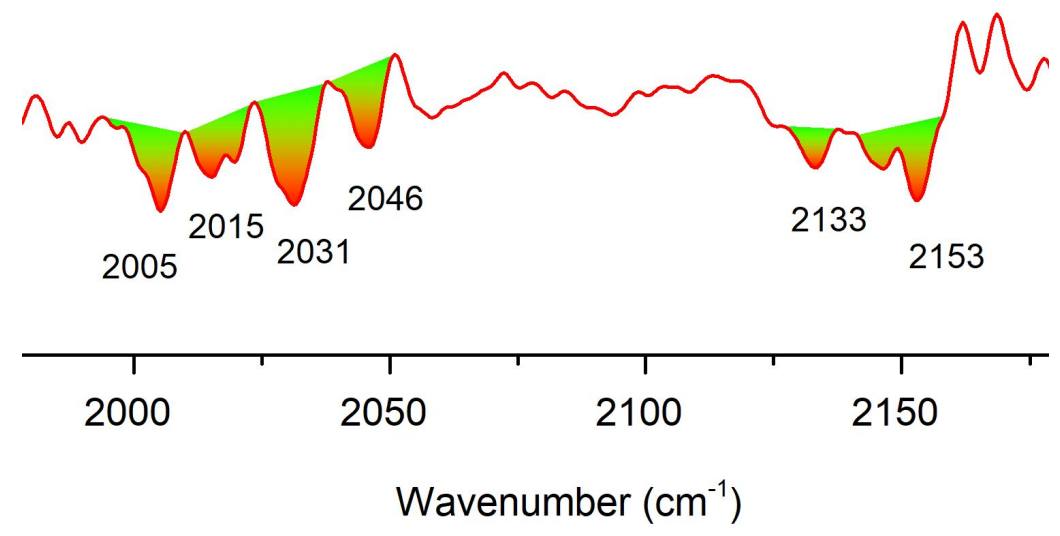

Figure S7. The attenuated total refraction Fourier transform infrared spectroscopy (ATR-FTIR) of fresh and spent $\mathrm{Rh}_{1} / \mathrm{AC}$ catalysts. 
a

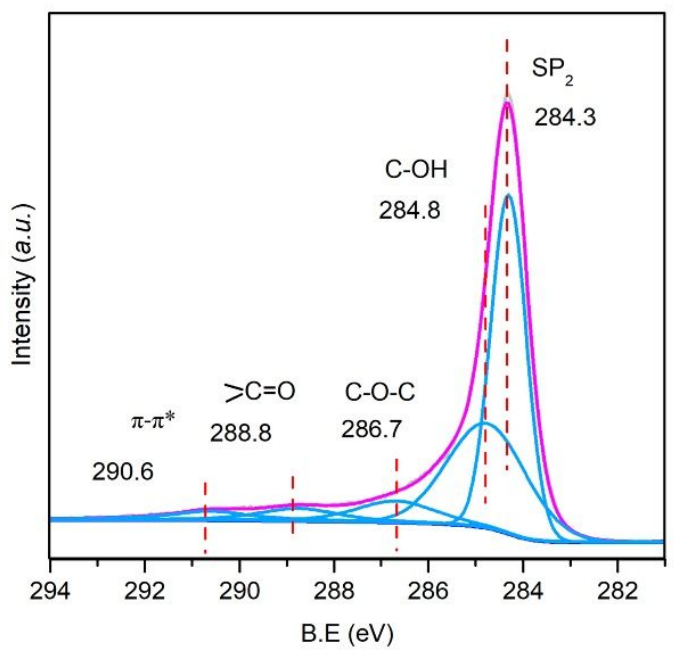

C

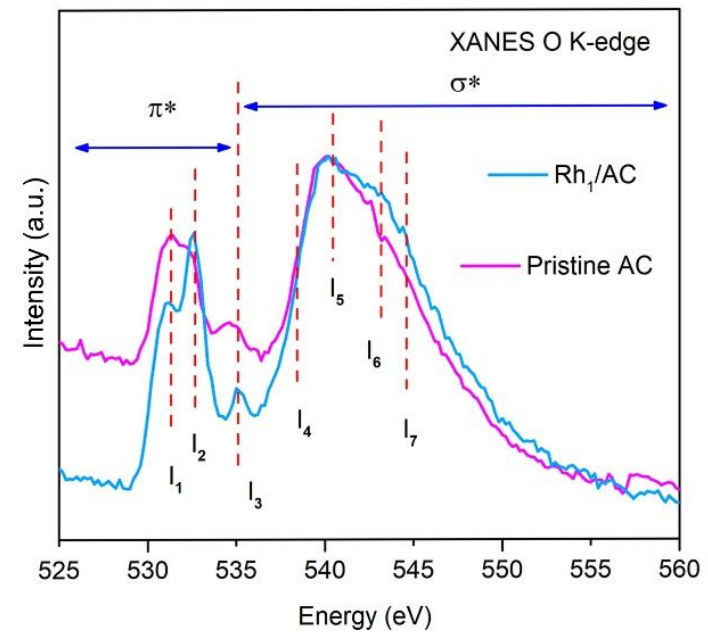

b

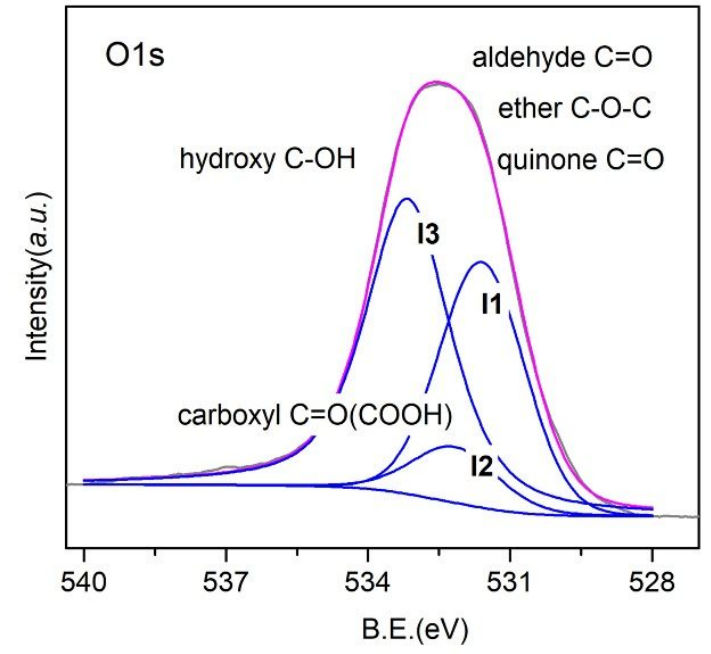

d

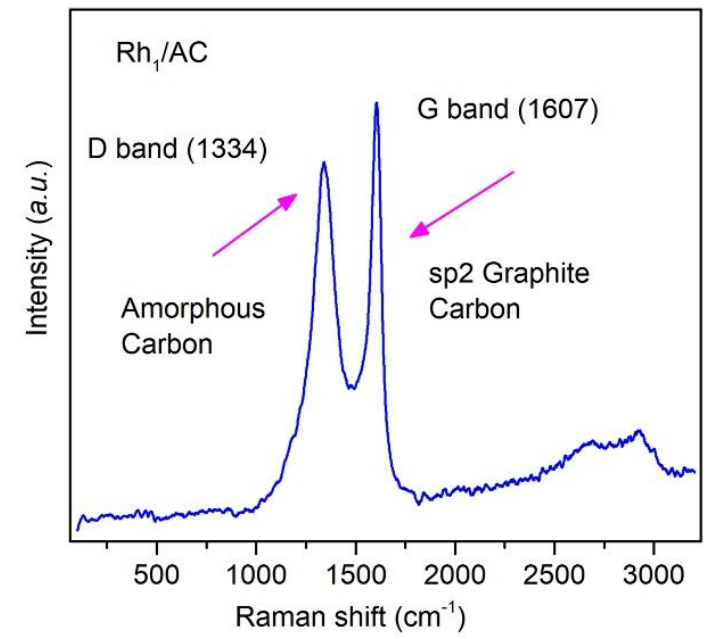

Figure S8. (a) C1s XPS of fresh $\mathrm{Rh}_{1} / \mathrm{AC}$, (b) O1s XPS of fresh $\mathrm{Rh}_{1} / \mathrm{AC}$, (c) $\mathrm{O} K$-edge XANES of AC support and fresh $\mathrm{Rh}_{1} / \mathrm{AC}\left(\mathrm{I}_{1}: \pi *(\mathrm{COOH}) ; \mathrm{I}_{2}: \pi *(\mathrm{C}-\mathrm{OH}) ; \mathrm{I}_{3}: \pi *\right.$ $(>\mathrm{O}=\mathrm{O})+\pi *(\mathrm{C}-\mathrm{O}-\mathrm{C}) ; \mathrm{I}_{4}: \sigma *(\mathrm{COOH}) ; \mathrm{I}_{5}: \sigma *(\mathrm{C}-\mathrm{OH}) ; \mathrm{I}_{6}: \sigma *(\mathrm{C}-\mathrm{O}-\mathrm{C}) ; \mathrm{I}_{7}: \sigma *(>\mathrm{O}=\mathrm{O})$, (d) AFM-Raman spectrum of fresh $\mathrm{Rh}_{1} / \mathrm{AC}$ catalyst. 


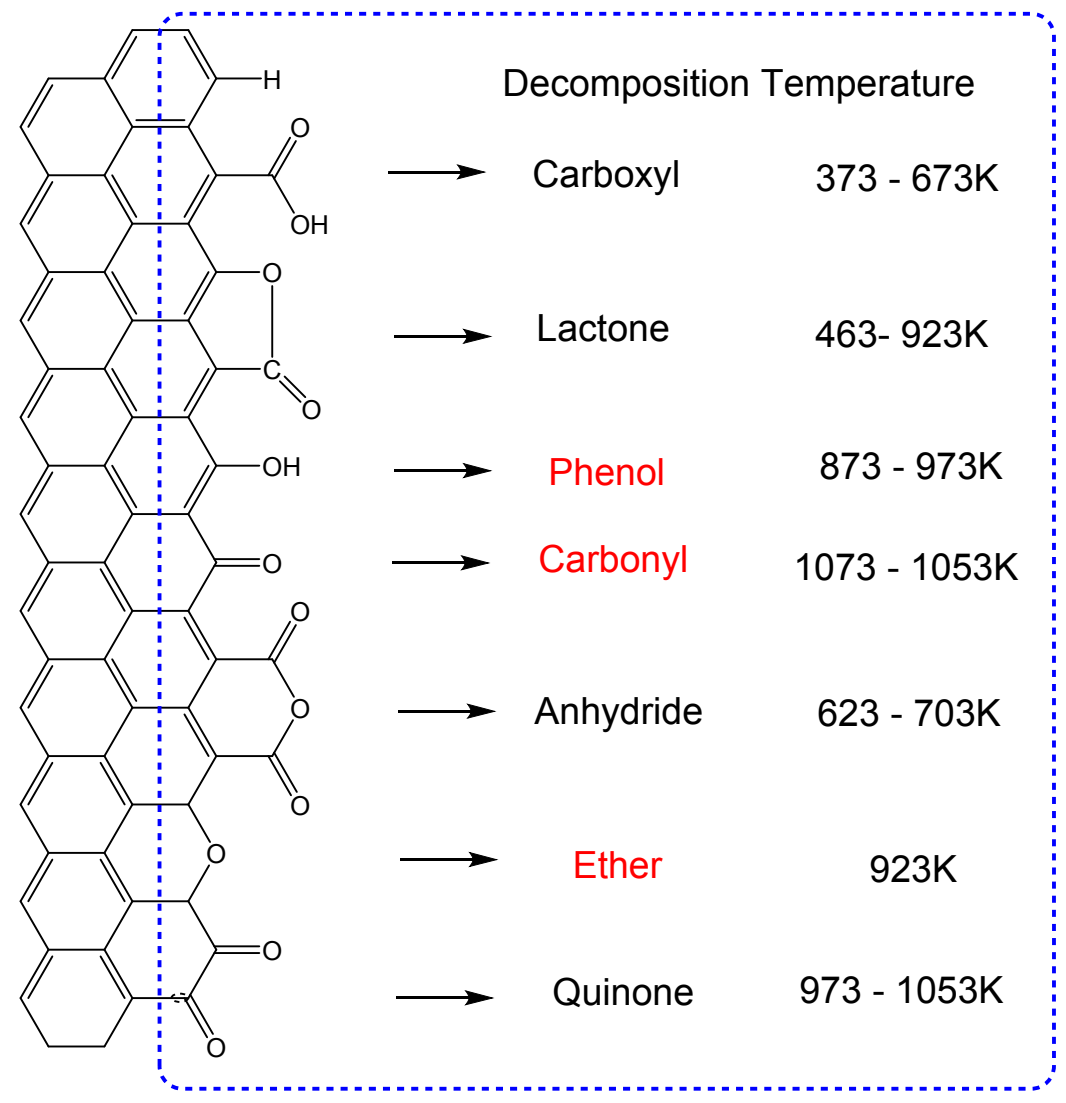

Figure S9. The thermal stability of surface oxygen-containing groups on activated carbon $^{1-2}$. 
a

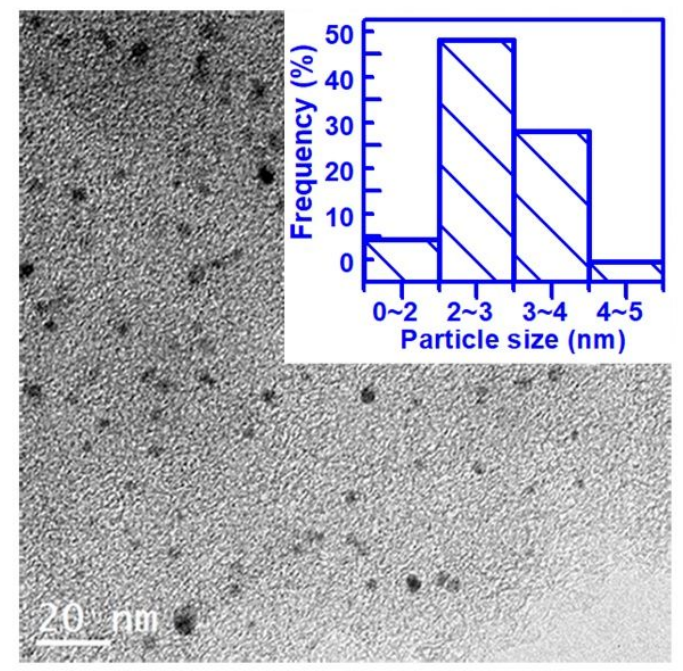

b

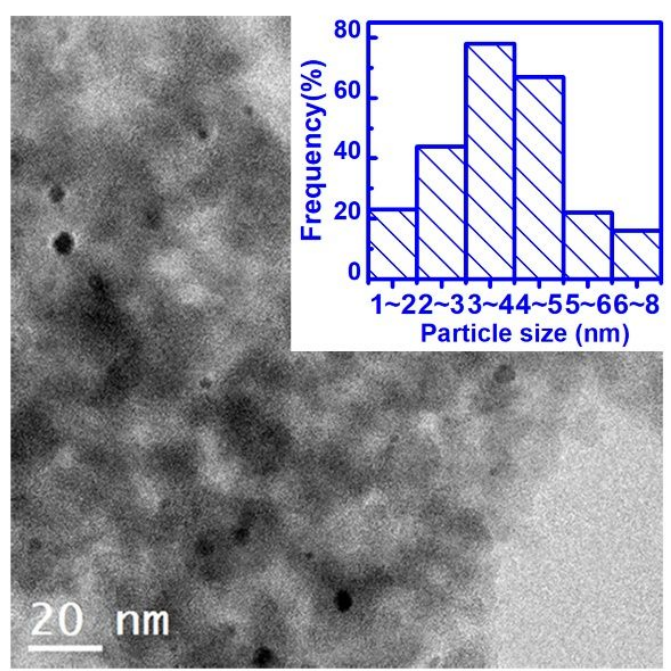

Figure S10. TEM image of (a) fresh $\mathrm{Rh} / \mathrm{AC}$ and (b) $\mathrm{Rh} / \mathrm{SiO}_{2}$ catalysts. 


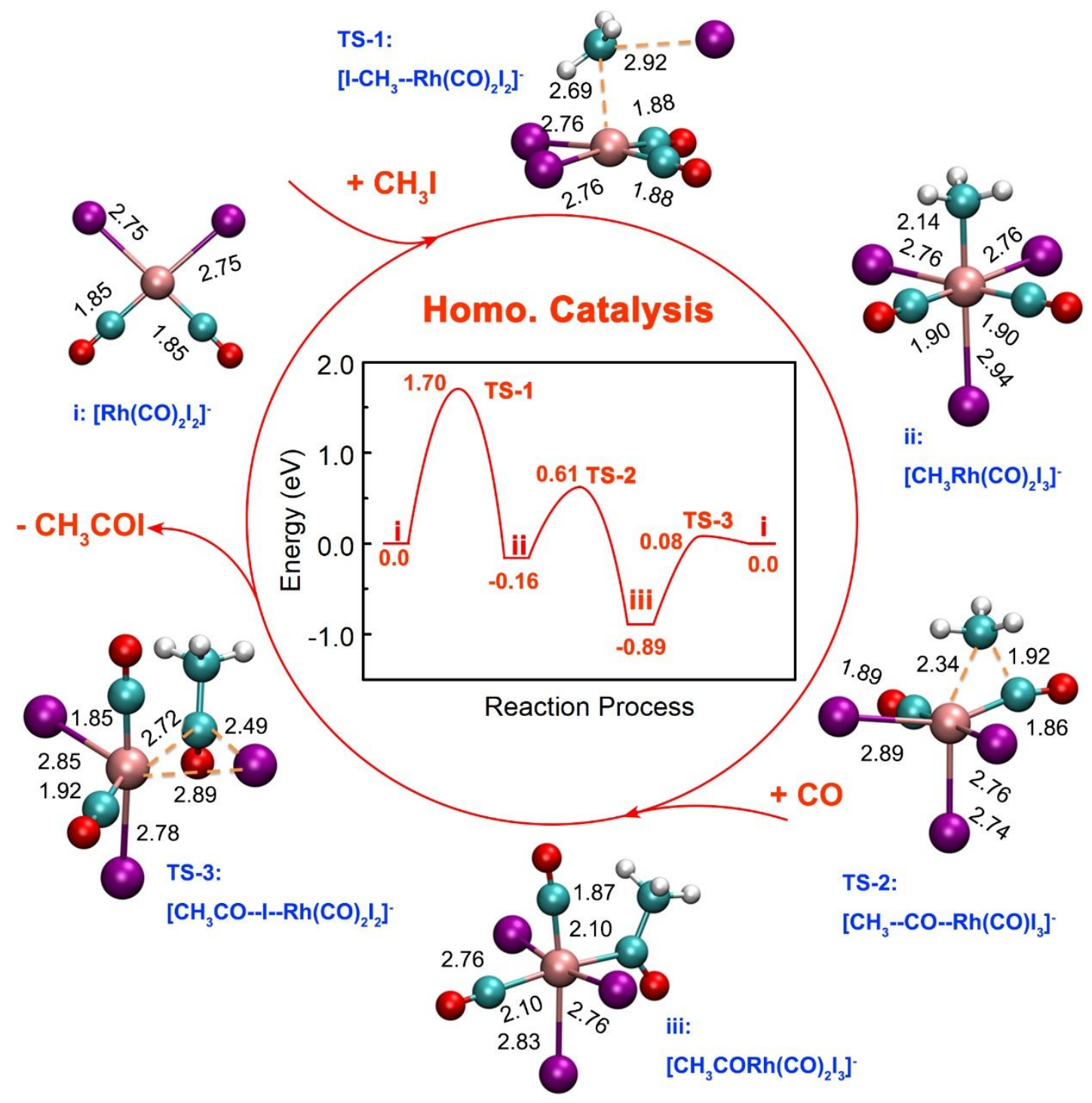

Figure S11. DFT calculated energy barrier of homogeneous methanol carbonylation reaction process based on B3LYP-D3 method with $6-31+\mathrm{g} * *$ for $\mathrm{C}, \mathrm{H}, \mathrm{O}$ and other atoms for def2-TZVPD. Here, the solvent effect for homogeneous environment was considered with the solvent model chosen to be Polarizable Continuum Model (PCM) using CPCM method. 


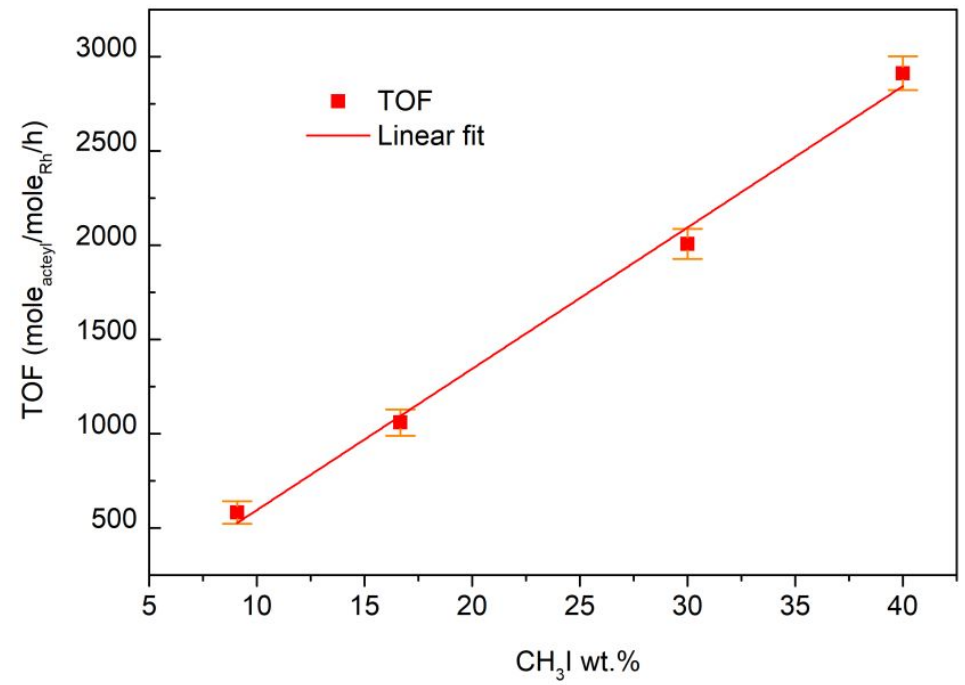

Figure S12. Effect of mass ratio of $\mathrm{CH}_{3} \mathrm{I}$ on activity of $\mathrm{Rh}_{1} / \mathrm{AC}$ catalyst. 
Table S1. The fitting data of C1s XPS of $\mathrm{Rh}_{1} / \mathrm{AC}$ catalyst

\begin{tabular}{cccccc}
\hline C 1s & $\mathrm{C}_{1}$ & $\mathrm{C}_{2}$ & $\mathrm{C}_{3}$ & $\mathrm{C}_{4}$ & $\mathrm{C}_{5}$ \\
\hline Peak B.E (eV) & 284.3 & 284.8 & 286.7 & 288.8 & 290.6 \\
\hline Area\% & 49.0 & 33.9 & 7.8 & 5.0 & 4.3 \\
\hline FWHM $(\mathrm{eV})$ & 0.884 & 2.03 & 2.03 & 2.03 & 2.03 \\
\hline
\end{tabular}

$\mathrm{C}_{1}, \mathrm{C}_{2}, \mathrm{C}_{3}, \mathrm{C}_{4}, \mathrm{C}_{5}$ were attributed to $\mathrm{C}\left(\mathrm{SP}_{2}\right), \mathrm{C}-\mathrm{OH}, \mathrm{C}-\mathrm{O}-\mathrm{C}, \mathrm{C}=\mathrm{O}, \pi-\pi^{*}$, respectively (J. Phys. Chem. C 2011, 115, 17009-17019) 
Table S2. The fitting data of O1s XPS of $\mathrm{Rh}_{1} / \mathrm{AC}$ catalyst

\begin{tabular}{cccc}
\hline O 1s & $\mathrm{O}_{1}$ & $\mathrm{O}_{2}$ & $\mathrm{O}_{3}$ \\
\hline Peak B.E $(\mathrm{eV})$ & 531.6 & 532.2 & 533.2 \\
\hline Area\% & 36.5 & 8.8 & 54.7 \\
\hline FWHM $(\mathrm{eV})$ & 2.11 & 2.11 & 2.11 \\
\hline
\end{tabular}

$\mathrm{O}_{1}$ was mainly attributed to $-\mathrm{C}=\mathrm{O}$ and $\mathrm{C}-\mathrm{O}-\mathrm{C} ; \mathrm{O}_{2}$ was ascribed to carboxylic (-COOH), lactone; $\mathrm{O}_{3}$ was belonged as $\mathrm{C}-\mathrm{OH}$, like phenolic hydroxyl. 
Table S3. Catalytic activity comparison of the $\mathrm{Rh}_{1} / \mathrm{AC}$ with the commercial homogeneous catalysts and other supported catalysts for methanol carbonylaton

\begin{tabular}{|c|c|c|c|c|c|}
\hline Catalysts & Process & $\begin{array}{c}\text { Catalytic active } \\
\text { site }\end{array}$ & $\begin{array}{c}\text { STY } \\
\left(\mathrm{g} / \mathrm{kg}_{\mathrm{cat}} / \mathrm{h}\right)\end{array}$ & $\operatorname{TOF}\left(\mathrm{h}^{-1}\right)$ & Promoter \\
\hline $1 \mathrm{wt} \% \mathrm{Rh}_{1} / \mathrm{AC}$ & This work & Single site $\mathrm{Rh}$ & 19100 & 3133 & $\mathrm{CH}_{3} \mathrm{I}$ \\
\hline$\left[\mathrm{Rh}(\mathrm{CO})_{2} \mathrm{I}_{2}\right]^{-}$ & Monsanto & Single site $\mathrm{Rh}$ & - & $1465^{3-4}$ & $\mathrm{CH}_{3} \mathrm{I}$ \\
\hline $1 \mathrm{wt} \% \operatorname{Ir}_{1} / \mathrm{AC}$ & Ref.[4] & Single site Ir & 7400 & $2000^{4}$ & $\mathrm{CH}_{3} \mathrm{I}$ \\
\hline $\mathrm{BP}\left[\operatorname{Ir}(\mathrm{CO})_{2} \mathrm{I}_{2}\right]^{-}$ & Cativa $^{\mathrm{TM}}$ & Single site Rh & - & $1595^{5}$ & $\mathrm{CH}_{3} \mathrm{I}$ \\
\hline $\begin{array}{c}1 \mathrm{wt} \% \mathrm{Ir} \\
\mathrm{Ir}_{1}-\mathrm{La}_{1} / \mathrm{AC}\end{array}$ & Ref.[6] & Single site Ir & 11180 & $3600^{6}$ & $\mathrm{CH}_{3} \mathrm{I}$ \\
\hline Acetica Rh/PVPs & $\begin{array}{c}\text { Chiyoda \& } \\
\text { UOP }\end{array}$ & Single site $\mathrm{Rh}$ & $6000^{7}$ & $1723^{3-4,8}$ & $\mathrm{CH}_{3} \mathrm{I}$ \\
\hline $0.03 \% \mathrm{Rh} / \mathrm{Y}$ Zeolite & Ref.[9] & Single site $\mathrm{Rh}$ & - & $2646^{9}$ & $\mathrm{CH}_{3} \mathrm{I}$ \\
\hline $1 \% \operatorname{Ir} / \mathrm{AC}$ & Ref.[10] & Nano metal Ir & $3500^{10}$ & - & $\mathrm{CH}_{3} \mathrm{I}$ \\
\hline $5.53 \% \mathrm{Ni} / \mathrm{AC}$ & $\begin{array}{c}\text { Ref.[11,12 } \\
]\end{array}$ & Nano metal Ni & $3040^{11-12}$ & - & $\mathrm{CH}_{3} \mathrm{I}$ \\
\hline $1.3 \% \mathrm{Cu} / \mathrm{MOR}$ & Ref.[13] & Nano metal $\mathrm{Cu}$ & $156^{13}$ & - & - \\
\hline $\mathrm{Ir}-\mathrm{La} / \mathrm{C}$ & Ref.[14] & Single site Ir & - & $5400^{14}$ & $\mathrm{CH}_{3} \mathrm{I}$ \\
\hline $\begin{array}{c}0.265 \% \\
\text { Rh/POL-2BPY }\end{array}$ & Ref.[15] & Single site $\mathrm{Rh}$ & - & $1500^{15}$ & $\mathrm{CH}_{3} \mathrm{I}$ \\
\hline $\mathrm{Rh} / \mathrm{SiO}_{2}$ & Ref.[16] & Single site $\mathrm{Rh}$ & - & $0^{16}$ & - \\
\hline $0.2 \% \mathrm{Rh}-5 \% \mathrm{Na} / \mathrm{ZrO}_{2}$ & Ref.[17] & Single site $\mathrm{Rh}$ & - & $37^{17}$ & - \\
\hline $10 \% \mathrm{Re} / \mathrm{SiO}_{2}$ & Ref.[16] & Single site Re & - & $13.4^{16}$ & - \\
\hline
\end{tabular}


Table S4. ICP-OES of fresh and spent $\mathrm{Rh}_{1} / \mathrm{AC}$ catalysts

\begin{tabular}{lll}
\hline Samples & Fresh $\mathrm{Rh}_{1} / \mathrm{AC}$ & Spent $\mathrm{Rh}_{1} / \mathrm{AC}$ \\
\hline ICP-OES g/gcat $(\%)$ & 0.978 & 0.956 \\
\hline
\end{tabular}


Table S5. Activity comparison of $\mathrm{Rh}_{1} / \mathrm{AC}, \mathrm{Rh} / \mathrm{SiO}_{2}$, and $\left[\mathrm{Rh}(\mathrm{CO})_{2} \mathrm{I}_{2}\right]^{-}$catalyst for methanol carbonylation reaction

\begin{tabular}{|c|c|c|c|c|c|c|c|c|}
\hline \multirow{2}{*}{ Catalysts } & \multirow{2}{*}{$\begin{array}{l}\text { Conv.\% } \\
\mathrm{CH}_{3} \mathrm{OH}\end{array}$} & \multirow{2}{*}{$\begin{array}{l}\text { Conv. } \% \\
\text { CO }\end{array}$} & \multicolumn{3}{|c|}{ Sel.\% } & \multirow{2}{*}{$\begin{array}{c}\text { TOF } \\
\left(\text { mole }_{\text {acetyl }} /\right. \\
\left.\text { mole }_{\mathrm{Rh}} \cdot \mathrm{h}\right)\end{array}$} & \multirow{2}{*}{$\begin{array}{c}\text { STY } \\
(\text { gacetyls } / \mathrm{kgcat} \cdot \mathrm{h})\end{array}$} & \multirow{2}{*}{$\begin{array}{c}\text { Carbon } \\
\text { balance } \\
(\mathrm{C} \%)\end{array}$} \\
\hline & & & AA & MA & $\mathrm{CH}_{4}$ & & & \\
\hline $\mathrm{Rh}_{1} / \mathrm{AC}^{*}$ & 95.4 & 52.5 & 37.5 & 58.3 & 4.2 & 2976 & 18500 & 93.4 \\
\hline$\left[\mathrm{Rh}(\mathrm{CO})_{2} \mathrm{I}_{2}\right]^{-}$ & 83.7 & 34.7 & 32.7 & 54.7 & 0.7 & 1059 & - & 87.4 \\
\hline $\mathrm{Rh}_{1} / \mathrm{AC}$ & 99.8 & 65.0 & 18.3 & 72.6 & 9.1 & 3133 & 19100 & 93.3 \\
\hline $\mathrm{Rh} / \mathrm{AC}^{*}$ & 10.5 & 4.2 & 5.2 & 89.7 & 5.1 & 100 & 724 & 92.4 \\
\hline $\mathrm{Rh} / \mathrm{SiO}_{2}$ & 0.3 & 0.1 & 0.0 & 81.5 & 18.5 & 10 & 47 & 105.7 \\
\hline $\mathrm{Rh}_{1} / \mathrm{AC}^{* *}$ & 96.0 & 53.2 & 37.5 & 57.4 & 4.8 & 3015 & 18700 & 95.6 \\
\hline
\end{tabular}

AA and MA represent the products of acetyl species $\mathrm{CH}_{3} \mathrm{COOH}$ and $\mathrm{CH}_{3} \mathrm{COOCH}_{3}$, respectively. Mass balance was calculated based on methanol carbon numbers $(\mathrm{C} \%)$ in the reactants and products. Error was allowed in the range of $\pm 5 \sim 10 \%$.

Reaction conditions: $\mathrm{Rh}_{1} / \mathrm{AC}^{*}, 463 \mathrm{~K}, 2.8 \mathrm{MPa}, \mathrm{CO}=55.4 \mathrm{ml} / \mathrm{min}, \mathrm{H}_{2}=5.5 \mathrm{ml} / \mathrm{min}$, $\mathrm{CH}_{3} \mathrm{OH}_{\text {(Liquid) }}=87.03 \mathrm{uL} / \mathrm{min}, \mathrm{CH}_{3} \mathrm{I}_{\text {(Liquid) }}=12.97 \mathrm{uL} / \mathrm{min} ;\left[\mathrm{Rh}(\mathrm{CO})_{2} \mathrm{I}_{2}\right]^{-}, 463 \mathrm{~K}, 2.8 \mathrm{MPa}$, reaction time $2.0 \mathrm{~h}$, reaction time $=20 \mathrm{~min}, \quad m\left(\mathrm{CH}_{3} \mathrm{OH}\right)=55.4 \mathrm{~g}, m\left(\mathrm{CH}_{3} \mathrm{I}\right)=24.0 \mathrm{~g}, m$ $\left(\mathrm{H}_{2} \mathrm{O}\right)=20.0 \mathrm{~g}$, cat: $\mathrm{Rh}_{2}(\mathrm{CO})_{4} \mathrm{Cl}_{2}, 0.025 \mathrm{~g} . \mathrm{Rh}_{1} / \mathrm{AC}, \mathrm{Rh} / \mathrm{AC}^{*}$ and $\mathrm{Rh} / \mathrm{SiO}_{2}, 513 \mathrm{~K}, 1.7$ $\mathrm{MPa}, \quad \mathrm{CO}=55.4 \mathrm{ml} / \mathrm{min}, \quad \mathrm{H}_{2}=5.5 \mathrm{ml} / \mathrm{min}, \quad \mathrm{CH}_{3} \mathrm{OH}_{\text {(Liquid) }}=87.03 \mathrm{uL} / \mathrm{min}$, $\mathrm{CH}_{3} \mathrm{I}_{\text {(Liquid) }}=12.97 \mathrm{uL} / \mathrm{min} ; \mathrm{Rh}_{1} / \mathrm{AC}^{* *}$ denoted the data after $100 \mathrm{~h}$ time on stream for the $\mathrm{Rh}_{1} / \mathrm{AC}$ catalyst. The data of $\mathrm{Rh}_{1} / \mathrm{AC}, \mathrm{Rh} / \mathrm{AC}^{*}$ and $\mathrm{Rh} / \mathrm{SiO}_{2}$ is obtained after $5 \mathrm{~h}$ time on stream. 
Table S6. Temperature effect on catalytic activity of $\mathrm{Rh}_{1} / \mathrm{AC}$ catalyst for heterogeneous methanol carbonylation reaction

\begin{tabular}{|c|c|c|c|c|c|c|c|c|}
\hline \multirow{2}{*}{$\mathrm{T} / \mathrm{K}$} & \multirow{2}{*}{$\begin{array}{l}\text { Conv.\% } \\
\mathrm{CH}_{3} \mathrm{OH}\end{array}$} & \multirow{2}{*}{$\begin{array}{c}\text { Conv. } \% \\
\text { CO }\end{array}$} & \multicolumn{3}{|c|}{ Sel.\% } & \multirow{2}{*}{$\begin{array}{c}\text { TOF } \\
\left(\text { mole }_{\text {acetyl }} /\right. \\
\left.\operatorname{mole}_{\mathrm{Rh}} \cdot \mathrm{h}\right)\end{array}$} & \multirow{2}{*}{$\begin{array}{c}\mathrm{STY} \\
\left(\mathrm{g}_{\text {acetyls }} / \mathrm{kg}_{\text {cat }} \cdot \mathrm{h}\right)\end{array}$} & \multirow{2}{*}{$\begin{array}{c}\text { Carbon } \\
\text { balance } \\
(\mathrm{C} \%)\end{array}$} \\
\hline & & & AA & MA & $\mathrm{CH}_{4}$ & & & \\
\hline 463 & 38.4 & 17.9 & 4.6 & 94.9 & 0.6 & 1095 & 7700 & 93.9 \\
\hline 453 & 25.3 & 10.8 & 0.8 & 98.1 & 1.1 & 658 & 4700 & 89.7 \\
\hline 443 & 12.9 & 5.7 & 0.2 & 99.7 & 0.1 & 347 & 2500 & 92.2 \\
\hline 433 & 7.6 & 3.3 & 0.3 & 99.6 & 0.1 & 199 & 1400 & 95.4 \\
\hline 413 & 4.8 & 2.2 & 0.5 & 99.4 & 0.2 & 133 & 1000 & 94.8 \\
\hline
\end{tabular}

Reaction conditions: cat: $\mathrm{Rh}_{1} / \mathrm{AC}, 0.25 \mathrm{~g}, 1.7 \mathrm{MPa}, \mathrm{CO}=55.4 \mathrm{ml} / \mathrm{min}, \mathrm{H}_{2}=5.5 \mathrm{ml} / \mathrm{min}$, $\mathrm{CH}_{3} \mathrm{OH}_{(\text {Liquid })}=96.64 \mathrm{uL} / \mathrm{min}, \mathrm{CH}_{3} \mathrm{I}_{\text {(Liquid) }}=3.36 \mathrm{uL} / \mathrm{min}$. AA and $\mathrm{MA}$ represent the products of acetyl species $\mathrm{CH}_{3} \mathrm{COOH}$ and $\mathrm{CH}_{3} \mathrm{COOCH}_{3}$, respectively. Mass balance was calculated based on methanol carbon numbers $(\mathrm{C} \%)$ in the reactants and products. Error was allowed in the range of $\pm 5 \sim 10 \%$. 
Table S7. Temperature effect on catalytic activity of $\left[\mathrm{Rh}(\mathrm{CO})_{2} \mathrm{I}_{2}\right]^{-}$catalyst for homogeneous methanol carbonylation reaction

\begin{tabular}{|c|c|c|c|c|c|c|}
\hline \multirow{2}{*}{$\mathrm{T} / \mathrm{K}$} & \multirow{2}{*}{$\begin{array}{l}\text { Conv.\% } \\
\mathrm{CH}_{3} \mathrm{OH}\end{array}$} & \multirow{2}{*}{$\begin{array}{c}\text { Conv. } \% \\
\text { CO }\end{array}$} & \multicolumn{2}{|c|}{ Sel.\% } & \multirow{2}{*}{$\begin{array}{c}\text { TOF } \\
\left(\text { mole }_{\text {acetyl }} /\right. \\
\left.\text { mole }_{\mathrm{Rh}} \cdot \mathrm{h}\right)\end{array}$} & \multirow{2}{*}{$\begin{array}{c}\text { Carbon } \\
\text { balance } \\
(\mathrm{C} \%)\end{array}$} \\
\hline & & & AA & MA & & \\
\hline 453 & 23.7 & 12.7 & 53.3 & 46.7 & 1133 & 79.7 \\
\hline 443 & 11.1 & 7.7 & 84.0 & 16.0 & 439 & 81.0 \\
\hline 433 & 7.5 & 4.9 & 87.1 & 22.9 & 179 & 85.0 \\
\hline
\end{tabular}

Reaction conditions: cat: $\mathrm{Rh}_{2}(\mathrm{CO})_{4} \mathrm{Cl}_{2}, 0.025 \mathrm{~g}, \mathrm{P}_{\mathrm{CO} / \mathrm{H} 2}=1.0 \mathrm{MPa}, \mathrm{CO} / \mathrm{H}_{2}=10$, reaction time $=20 \mathrm{~min}, \mathrm{~m}\left(\mathrm{CH}_{3} \mathrm{OH}\right)=55.4 \mathrm{~g}, m\left(\mathrm{CH}_{3} \mathrm{I}\right)=24.0 \mathrm{~g}, m\left(\mathrm{H}_{2} \mathrm{O}\right)=20.0 \mathrm{~g}$. AA and MA represent the products of acetyl species $\mathrm{CH}_{3} \mathrm{COOH}$ and $\mathrm{CH}_{3} \mathrm{COOCH}_{3}$, respectively. Mass balance was calculated based on methanol carbon numbers $(\mathrm{C} \%)$ in the reactants and products. Error was allowed in the range of $\pm 10 \sim 20 \%$, due to the formation dimethyl ether, which is liquid above $0.5 \mathrm{MPa}$, but in gas state in the ambient atmosphere. 


\section{References:}

1. Figueiredo, J. L.; Pereira, M. F. R.; Freitas, M. M. A.; Órfão, J. J. M., Modification of the surface chemistry of activated carbons. Carbon 1999, 37 (9), 1379-1389.

2. Kwiatkowski, J. F., Activated carbon : classifications, properties and applications. Nova Science Publishers: 2012.

3. Smith, B. L.; Torrence, G. P.; Murphy, M. A.; Aguiló, A., The rhodiumcatalyzed methanol carbonylation to acetic acid at low water concentrations: the effect of iodide and acetate on catalyst activity and stability. J. Mol. Catal. 1987, 39 (1), $115-136$

4. Feng, S.; Lin, X.; Song, X.; Liu, Y.; Jiang, Z.; Hemberger, P.; Bodi, A.; Ding, Y., The role of $\mathrm{H}_{2}$ on the stability of the single-metal-site $\operatorname{Ir} 1$ /AC catalyst for heterogeneous methanol carbonylation. J. Catal. 2020, 381, 193-203.

5. Sunley, G. J.; Watson, D. J., High productivity methanol carbonylation catalysis using iridium - The Cativa (TM) process for the manufacture of acetic acid. Catal. Today 2000, 58 (4), 293-307.

6. Feng, S.; Lin, X.; Song, X.; Liu, Y.; Jiang, Z.; Ding, Y., Insight into the stability of binuclear $\mathrm{Ir}-\mathrm{La}$ catalysts for efficient heterogeneous methanol carbonylation. $J$. Catal. 2019, 377, 400-408.

7. Feng, S.; Song, X.; Ren, Z.; Ding, Y., La-stabilized, single-atom Ir/AC catalyst for heterogeneous methanol carbonylation to methyl acetate. Ind. Eng. Chem. Res. 2019, 58 (12), 4755-4763. 
8. Noriyuki, Y.; Takeshi, M.; Joe, W.; Ben, S. In Studies in Surface Science and Catalysis, Hattori, H., Otsuka, K., Eds. Elsevier: 1999; Vol. 121, pp 93-98.

9. Yashima, T.; Orikasa, Y.; Takahashi, N.; Hara, N., Vapor phase carbonylation of methanol over RhY zeolite. J. Catal. 1979, 59 (1), 53-60.

10. Zehl, G.; Bischoff, S.; Lücke, B., Vapor-phase carbonylation of methanol on an active carbon supported iridium-catalyst. Catal. Lett. 1993, 19 (2-3), 247-255.

11. Pang, F.; Song, F.; Zhang, Q.; Tan, Y.; Han, Y., Study on the influence of oxygen-containing groups on the performance of $\mathrm{Ni} / \mathrm{AC}$ catalysts in methanol vapor-phase carbonylation. Chem. Eng. J. 2016, 293, 129-138.

12. Fujimoto, K.; Shikada, T.; Omata, K.; Tominaga, H.-O., Vapor phase carbonylation of methanol with supported nickel metal catalysts. Ind. Eng. Chem. Res. 1982, $21(3), 429-432$.

13. Zhan, H.; Huang, S.; Li, Y.; Lv, J.; Wang, S.; Ma, X., Elucidating the nature and role of $\mathrm{Cu}$ species in enhanced catalytic carbonylation of dimethyl ether over Cu/H-MOR. Catal. Sci. Technol. 2015, 5 (9), 4378-4389.

14. Kwak, J. H.; Dagle, R.; Tustin, G. C.; Zoeller, J. R.; Allard, L. F.; Wang, Y., Molecular active sites in heterogeneous $\mathrm{Ir}-\mathrm{La} / \mathrm{C}$-catalyzed carbonylation of methanol to acetates. J. Phys. Chem. Lett. 2014, 5 (3), 566-572.

15. Ren, Z.; Liu, Y.; Lyu, Y.; Song, X.; Zheng, C.; Feng, S.; Jiang, Z.; Ding, Y., Single-atom Rh based bipyridine framework porous organic polymer: A high active and superb stable catalyst for heterogeneous methanol carbonylation. J. Catal. 2019, $369,249-256$. 
16. Ji, Q.; Jordan, F.; Hossein, R.; Mingjie, X.; Adam, S. H.; Simon, R. B.; Xiaoqing, P.; Phillip, C., Selective Methanol Carbonylation to Acetic Acid on Heterogeneous Atomically Dispersed $\mathrm{ReO}_{4} / \mathrm{SiO}_{2}$ Catalysts. J. Am. Chem. Soc. 2020, $142(33), 14178-14189$.

17. Ji, Q.; Phillip, C.; Atomically dispersed Rh active sites on oxide supports with controlled acidity for gas-phase halide-free methanol carbonylation to acetic acid. Ind. Eng. Chem. Res. 2019, 58 (28). 